\title{
EFFICACY OF PBO-FRCM STRENGTHENING OF RC COLUMNS IN MRFS
}

\author{
A. Monaco $^{1}$ and P. Colajanni ${ }^{2}$ \\ ${ }^{1}$ Dept. of Architecture and Design - Politecnico di Torino \\ Viale Mattioli 39, 10125 Torino - Italy \\ e-mail: alessia.monaco@polito.it \\ ${ }^{2}$ Dept. of Civil, Environmental, Aerospace and Material Engineering - University of Palermo \\ Viale delle Scienze, 90128 Palermo - Italy \\ piero.colajanni@unipa.it
}

\begin{abstract}
Innovative materials and techniques are widespread used for the strengthening and rehabilitation of existing structures. Recent researches have been developed on new fiber reinforced composites in which epoxy resin is replaced by inorganic cementitious material. These kind of cement-based composite material is known as Fiber Reinforced Cementitious Matrices (FRCM) recently used also in combination with synthetic polymeric fibers named PBO.

The efficiency of this new confining system has been demonstrated by a large number of compression tests on concrete specimens while there are only few experimental researches on the behaviour of large scale specimens under external action able to simulate the actual behaviour of columns in MRFs.

This paper firstly presents the results of experimental tests on squared and rectangular specimens of medium-size concrete columns reinforced by PBO-FRCM subject to axial load; successively, an experimental campaign is performed on RC columns subjected to axial force and bending moment. Confined and unconfined columns, manufactured with different cross sections and concrete types, are tested.

The experimental tests on concrete columns and $R C$ pillars are reproduced through finite element models in which the behaviour of the confined concrete is simulated using the Concrete Damaged Plasticity model with properly adapted plastic parameters, according to recent studies available in the literature. The stress-strain law used in the model is deduced from a classical approach available in the literature for FRP confined concrete which is properly modified in order to take into account the efficiency reduction of FRCM with respect to FRP wraps.
\end{abstract}

Keywords: FRCM, Confinement, RC Columns, Experimental Tests, Numerical modeling. 


\section{INTRODUCTION}

The rehabilitation of existing structures is one of the most important topic addressed by the scientific community in the last decades [1-15]. Innovative materials and techniques are widespread used for the strengthening of structures. In particular, recent researches have been developed on new fiber reinforced composite materials in which epoxy resin is replaced by inorganic cementitious material. These kind of cement-based composites are known as Fiber Reinforced Cementitious Matrices (FRCM). Despite FRCM is usually used with glass, aramidic or carbon fibers, recently the performance of this confinement system has been improved by adopting a synthetic polymeric fiber named polyparaphenylene benzobisoxazole (PBO). The molecular structure of PBO is capable of establishing chemical bond with hydrated compounds in a special inorganic binder by means of a hydraulic reaction which helps improving the mechanical properties of the FRCM system.

The efficiency of this new confining system has been demonstrated by a large number of compression tests on concrete specimens with different cross section shapes and corner radius, confinement amount and concrete strength. By contrast, there are only few experimental researches concerning the behaviour of large scale specimens under external action able to simulate the actual behaviour of columns of moment resistant frames (MRFs).

In this framework, this paper presents the results of experimental tests on squared and rectangular specimens of medium-size concrete columns reinforced by PBO-FRCM subject to axial load with the aim of extending the experimental result database available in the literature on the topic. Furthermore, an experimental campaign is performed on ten RC columns subjected to axial force and bending moment and the results are here presented and discussed. The columns have square and rectangular cross section and they are manufactured using two different concrete strength. The specimens are unconfined or wrapped with two or four layer of textile reinforcement and, then, subject to axial load and bending moment in static regime.

The experimental tests on concrete columns and $\mathrm{RC}$ pillars are reproduced through finite element (FE) models in which the behaviour of the confined concrete is simulated using the well-known Concrete Damaged Plasticity (CDP) model with properly adapted plastic parameters, according to recent studies available in the literature [1]. The stress-strain law implemented in the CDP model is the one proposed by Spoelstra and Monti [2] for FRP confined concrete properly modified in order to take into account a certain efficiency reduction of FRCM with respect to classical FRP wraps [3].

\section{EXPERIMENTAL TESTS}

\subsection{Medium-size concrete specimens}

The experimental campaign was conducted on ten specimens presenting both squared and rectangular cross-section with four different concrete type mixtures. Preliminary tests were performed for obtaining the mechanical properties of the plain concrete. In particular, the average compressive strength and ultimate strain values, $\mathrm{f}_{\mathrm{c} 0}$ and $\varepsilon_{\mathrm{c} 0}$, were found for each concrete mixture: for mix $1\left(f_{c 0}-10\right), \mathrm{f}_{\mathrm{c} 0}=10.47 \mathrm{~N} / \mathrm{mm}^{2}$ and $\varepsilon_{\mathrm{c} 0}=2.05 \%$; for $\operatorname{mix} 2\left(f_{c 0}-14\right), \mathrm{f}_{\mathrm{c} 0}=14.44 \mathrm{~N} / \mathrm{mm}^{2}$ and $\varepsilon_{\mathrm{c} 0}=2.32 \%$; for mix $3\left(f_{c 0}-19\right), \mathrm{f}_{\mathrm{c} 0}=19.07 \mathrm{~N} / \mathrm{mm}^{2}$ and $\varepsilon_{\mathrm{c} 0}=2.04 \%$; for mix $4\left(f_{c 0}-22\right), \mathrm{f}_{\mathrm{c} 0}=$ $22.26 \mathrm{~N} / \mathrm{mm}^{2}$ and $\varepsilon_{\mathrm{c} 0}=2.09 \%$.

Ten medium-size concrete columns $600 \mathrm{~mm}$ high were manufactured with squared $(200 \mathrm{x}$ $200 \mathrm{~mm})$ and rectangular $(200 \times 400 \mathrm{~mm})$ cross-section and different corner radius $(\mathrm{R}=15 \mathrm{~mm}$ or $\mathrm{R}=30 \mathrm{~mm}$ ). The specimens are identified in Table 1 by a alphanumerical code in the format 


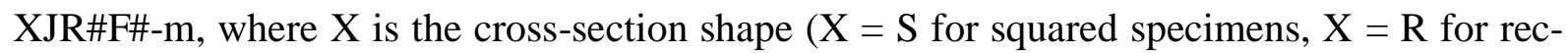
tangular specimens), $\mathrm{J}$ is the concrete type ( $\mathrm{J}=10$ for mix $f_{c 0}-10, \mathrm{~J}=14$ for mix $f_{c 0}-14, \mathrm{~J}=19$ for mix $f_{c 0}-19, \mathrm{~J}=22$ for mix $f_{c 0}-22$ ), R\# is the corner radius ( $\mathrm{R} 15$ for $15 \mathrm{~mm}$ and $\mathrm{R} 30$ for 30 $\mathrm{mm}$ ), F\# refers to the textile reinforcement ( $\mathrm{F} 0$ for the unconfined specimen, F\# for specimens confined with \# textile layers), finally the code letter $\mathrm{m}(\mathrm{m}=\mathrm{a}$ or $\mathrm{m}=\mathrm{b})$ is used to distinguish samples for identical specimens.

\begin{tabular}{|c|c|c|c|c|c|}
\hline ID & $\begin{array}{c}\text { cross-section } \\
{[\mathrm{mm} \times \mathrm{mm}]}\end{array}$ & $\begin{array}{c}\text { concrete } \\
\text { mix }\end{array}$ & $\begin{array}{c}\text { corner radius } \\
{[\mathrm{mm}]}\end{array}$ & $\begin{array}{c}\text { No. of textile } \\
\text { layers }\end{array}$ & $\begin{array}{c}\rho_{f} \\
{[\%]}\end{array}$ \\
\hline S19R15F0 & \multirow{4}{*}{$200 \times 200$} & & & 0 & - \\
\hline S19R15F2-a & & $\mathrm{f}_{\mathrm{c} 0^{-}}-19$ & 15 & \multirow{3}{*}{2} & \multirow{3}{*}{0.182} \\
\hline S19R15F2-b & & & & & \\
\hline $\begin{array}{l}\text { S14R30F2-a } \\
\text { S14R30F2-b }\end{array}$ & & $\mathrm{f}_{\mathrm{c} 0} \mathrm{l}^{-14}$ & 30 & & \\
\hline R10R30F2 & \multirow{5}{*}{$200 \times 400$} & $\mathrm{f}_{\mathrm{c} 0^{-}}-10$ & & \multirow{3}{*}{2} & \multirow{3}{*}{0.137} \\
\hline R14R30F2 & & $\mathrm{f}_{\mathrm{c} 0}-14$ & & & \\
\hline R19R30F2 & & $\mathrm{f}_{\mathrm{c} 0}-19$ & 30 & & \\
\hline R14R30F4 & & $\mathrm{f}_{\mathrm{c} 0}-14$ & & \multirow{2}{*}{4} & \multirow{2}{*}{0.273} \\
\hline R22R30F4 & & $\mathrm{f}_{\mathrm{c} 0-22}$ & & & \\
\hline
\end{tabular}

Table 1: Features of specimens.

Two or four PBO textile layers (Fig. 1) were applied to the columns obtaining the fiber volumetric ratios $\rho_{f}$ reported in Table 1 and calculated as $\rho_{f}=2 n t_{f}(b+h) /(b \cdot h)$, being $n$ the number of fiber layers, $t_{f}$ the nominal thickness of the fiber mesh, $b$ and $h$ the width and depth of the cross section. The specimens were wrapped for a length of $500 \mathrm{~mm}$, keeping unreinforced the portions of the columns at the top and bottom end for a length of $50 \mathrm{~mm}$ with the aim of avoiding a direct loading of the PBO-FRCM reinforcement during the test execution. The geometry of the wrapped columns is reported in Fig. 2.

The compression tests on all columns were carried out with a hydraulic press with a maximum load capacity of $4000 \mathrm{kN}$, which enables tests in controlled displacement mode (Fig. 3a). An electronic control unit with user interface via personal computer, regulated the test type and the equipment.

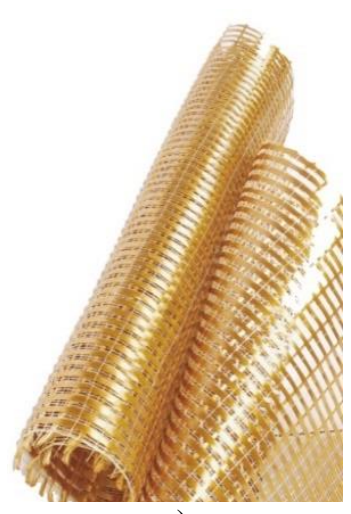

a)

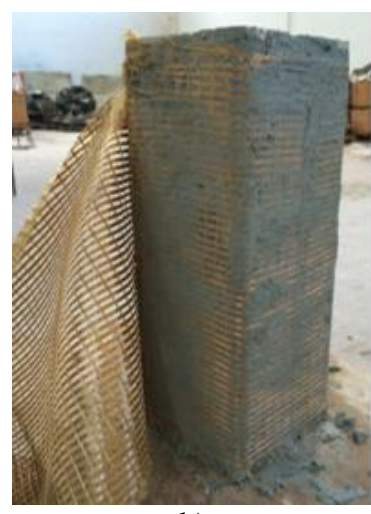

b)

Figure 1: Confining technique a) PBO fiber; b) application of PBO-FRCM wrapping to the specimen. 


\section{PBO-FRCM wrapping}

self levelling mortar
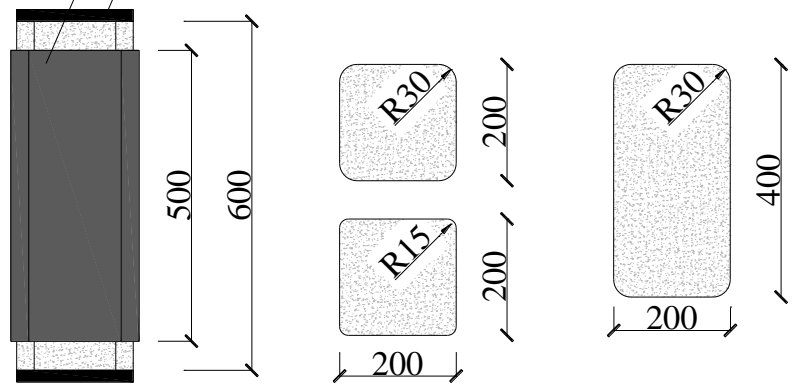

200

Figure 2: Geometry of the concrete specimens.

Two electronic gauges with gauge length $200 \mathrm{~mm}$ were installed on two parallel sides of each specimen in the axial direction at middle height for measuring the axial strains; conversely, for the measurement of the transversal strains, two further gauges with measure length of 100 $\mathrm{mm}$ were collocated on the other two sides (Fig. 3b). The loading of all specimens was conducted up to failure, adopting a rate displacement of $0.5 \mathrm{~mm} / \mathrm{s}$.

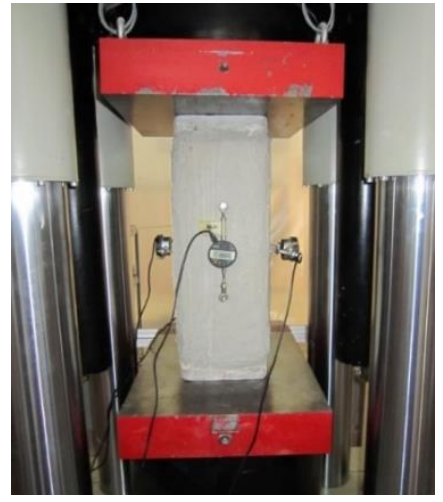

a)

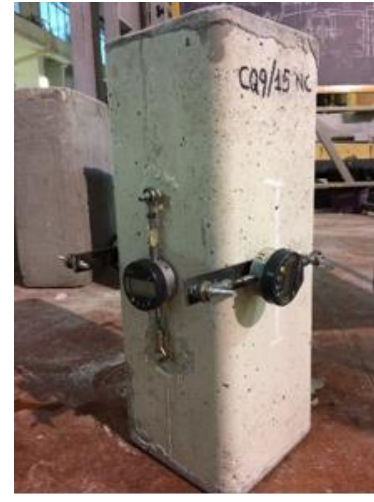

b)

Figure 3: Test setup a) specimen within the testing machine; b) position of gauges.

\subsection{Full scale RC columns}

Ten reinforced concrete $(\mathrm{RC})$ columns with square or rectangular cross section were tested. Specimens with two different concrete strength were manufactured, unconfined or wrapped with two or four layer of textile and then subject to axial load and bending moment.

The columns were cast with concrete types typical of buildings that require structural retrofitting (i.e. low-strength concrete). Portland cement (ASTM International Type I) was used. The cement : sand : gravel proportions in the concrete mixture were roughly 1:1.9:2.33 by weight and the maximum size of the coarse aggregate was $20 \mathrm{~mm}$. Two different water/cement ratios, namely $0.85,0.75$, where chosen in order to obtain two different low compressive concrete strengths. The average cylindrical compressive strengths, obtained by three cylindrical $150 \mathrm{~mm}$ $\mathrm{x} 300 \mathrm{~mm}$ specimens for each $\mathrm{w} / \mathrm{c}$ ratio, were $\mathrm{f}_{\mathrm{co}}=14.36 \mathrm{Mpa}$, and $\mathrm{f}_{\mathrm{co}}=18.98 \mathrm{Mpa}$, respectively. The Ruredil X Mesh Gold was used as PBO fibre textile. It is a bidirectional warped mesh, with four times as many fibres in the direction of the warp as in the direction of the weft. The equivalent textile thickness in the warp (longitudinal) direction was $0.045 \mathrm{~mm}$, while in the weft (transversal) direction it was $0.012 \mathrm{~mm}$. The elasticity modulus $\left(\mathrm{E}_{\mathrm{f}}\right)$, the tensile strength $\left(\mathrm{f}_{\mathrm{fu}}\right)$ 
and the ultimate value of axial strain $\left(\varepsilon_{\mathrm{fu} 0}\right)$ of the PBO fibres in each direction were provided by the fibre manufacturer and equal to $E_{\mathrm{f}}=270 \mathrm{GPa}, \mathrm{f}_{\mathrm{fu}}=5800 \mathrm{MPa}$ and $\varepsilon_{\mathrm{fu} 0}=21.5 \mathrm{~mm} / \mathrm{m}$, respectively. For the mortar, a stabilized inorganic matrix Ruredil X Mesh M750 designed to connect the PBO textile with the concrete substrate was utilized. Four specimens with square cross-section with side $a=200 \mathrm{~mm}$ and six rectangular having sides $\mathrm{a} \times \mathrm{b}=200 \times 400 \mathrm{~mm}$, all of them $1800 \mathrm{~mm}$ in height were casted. Square specimens where reinforced with four rebars of diameter $\phi_{1}=12 \mathrm{~mm}$ at the corner and rectangular one with six of $\phi_{1}=16 \mathrm{~mm}$ (Fig. 4), stirrup diameter $\phi_{\mathrm{w}}=6$ and $\phi_{\mathrm{w}}=8$ for square and rectangular specimens respectively, and stirrup spacing $s=120 \mathrm{~mm}$. Four specimens were wrapped with two layers and three with four layers of textile of height of $1000 \mathrm{~mm}$, placed as to left at the two end a $400 \mathrm{~mm}$ height zone unwrapped. The specimens are identified by a code in the format XC\#\#R JJ F\#, where X refers to the crosssection shape ( $\mathrm{X}=\mathrm{S}$ for square specimens, $\mathrm{X}=\mathrm{R}$ for rectangular specimens), the two digit $\mathrm{C \# \#}$ identifies the concrete compressive cylindrical strength, JJ refers to the corner curvature radius $(\mathrm{JJ}=15$ or $\mathrm{JJ}=30)$, the digit $\mathrm{F} \#$ refers to the number of layer of confinement textile. In Table 2 the characteristic of the ten specimens are summarized.

Aiming at reproducing the load condition of columns in a MRF loaded by seismic force (Fig.5a), firstly a constant axial load was applied to the specimen by an hydraulic actuator and a prestressing device, then a three points bending test were performed, in order to reproduce the incresing of the bending moment due to the seismic force (Fig. 5b).

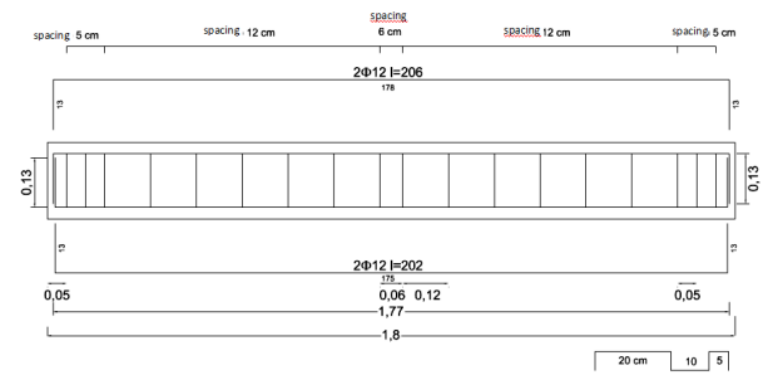

a)
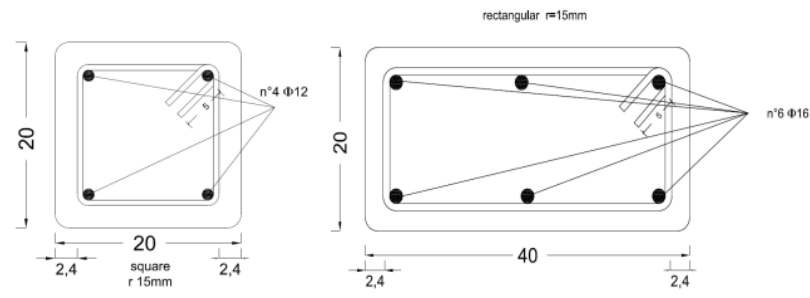

b)

Figure 4: RC specimens: a) longitudinal scheme; b) squared and rectangular cross sections.

\begin{tabular}{ccccc}
\hline Spec. & $\mathrm{Sec}$ & $\mathrm{f}_{\mathrm{cm}}$ & Corner & Fiber \\
\hline SC14R15F0 & $\mathrm{S}$ & 14 & 15 & 0 \\
$\mathrm{SC14R} 15 \mathrm{~F} 2$ & $\mathrm{~S}$ & 14 & 15 & 2 \\
$\mathrm{SC19R} 15 \mathrm{~F} 0$ & $\mathrm{~S}$ & 19 & 15 & 0 \\
$\mathrm{SC} 19 \mathrm{R} 30 \mathrm{~F} 2$ & $\mathrm{~S}$ & 19 & 30 & 2 \\
\hline $\mathrm{RC} 14 \mathrm{R} 15 \mathrm{~F} 0$ & $\mathrm{R}$ & 14 & 15 & 0 \\
$\mathrm{RC} 14 \mathrm{R} 15 \mathrm{~F} 4$ & $\mathrm{R}$ & 14 & 15 & 4 \\
$\mathrm{RC} 14 \mathrm{R} 30 \mathrm{~F} 4$ & $\mathrm{R}$ & 14 & 30 & 4 \\
$\mathrm{RC} 19 \mathrm{R} 15 \mathrm{~F} 0$ & $\mathrm{R}$ & 19 & 15 & 0 \\
$\mathrm{RC} 19 \mathrm{R} 15 \mathrm{~F} 2$ & $\mathrm{R}$ & 19 & 15 & 2 \\
$\mathrm{RC} 19 \mathrm{R} 15 \mathrm{~F} 4$ & $\mathrm{R}$ & 19 & 15 & 4 \\
\hline
\end{tabular}

Table 2: Full scale specimen details. 


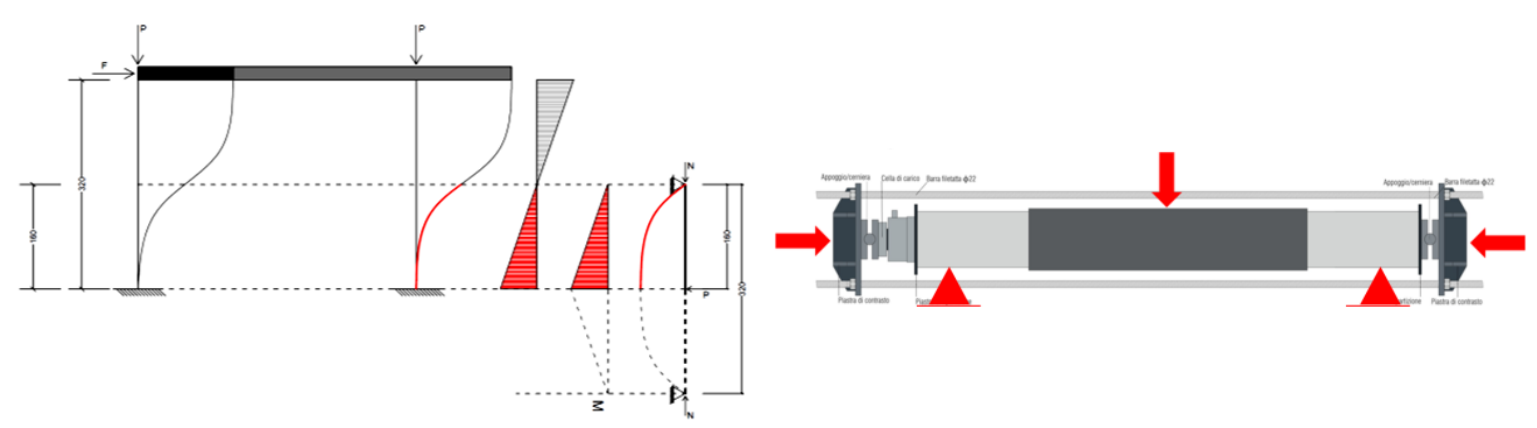

Figure 5: a) actual load condition and test load scheme; b) Test setup.

\section{NUMERCAL MODELING}

\subsection{Stress-strain laws for confined concrete}

The compressive behavior of confined concrete is modeled using analytical constitutive laws available in the literature. In particular, a modification of the model by Spoelstra and Monti [2] for fiber reinforced polymer (FRP) confined concrete is adopted. The stress-strain law $f_{c^{-}} \varepsilon_{c}$ of confined concrete is expressed as:

$$
f_{c}=\frac{f_{c c} \cdot x \cdot r}{r-1+x^{r}}
$$

where $x=\varepsilon_{d} \varepsilon_{c c} ; r=E_{d} /\left(E_{c}-E_{s e c}\right)$ with $E_{s e c}=f_{c c} / \varepsilon_{c c} ; \varepsilon_{c c}$ is the compressive axial strain at peak and $f_{c c}$ is the peak compressive strength. In this model, $f_{c c}$ is function of two parameters, i.e. the peak strength of plain concrete $f_{c 0}$ and the effective confining pressure $f_{l}$ :

$$
f_{c c}=f_{c 0}\left(2.254 \sqrt{1+7.94 \frac{f_{l}}{f_{c 0}}}-2 \frac{f_{l}}{f_{c 0}}-1.254\right)
$$

In case of passive confinement, the confining pressure increases continuously so that an incremental procedure is necessary for deriving the lateral strain $\varepsilon$ :

$$
\varepsilon_{l}\left(\varepsilon_{c}, f_{l}\right)=\frac{E_{c} \varepsilon_{c}-f_{c}\left(\varepsilon_{c}, f_{l}\right)}{2\left(5700 / \sqrt{f_{c 0}}-500\right) f_{c}\left(\varepsilon_{c}, f_{l}\right)}
$$

In order to take into account an efficiency reduction of FRCM with respect to classical FRP wraps, a coefficient $k_{f l, F R C M}$ is introduced in the model for calculating the confining pressure according to Colajanni et al. [3]:

$$
f_{l}=0.5 \cdot \rho \cdot E_{f} \cdot k_{l} \cdot \varepsilon_{l} \cdot k_{f l, F R C M}
$$

in which $\rho$ is the fiber volumetric ratio, $E_{f}$ is the elastic modulus of fibers and $k_{l}$ is a coefficient for rectangular sections according to CEB-FIP bulletin [4]. In particular, with reference to Fig. 6 , the coefficient of efficacy $\mathrm{k}_{1}$ is defined as:

$$
k_{l}=1-\frac{\left[\left(h-2 R_{c}\right)^{2}+\left(b-2 R_{c}\right)^{2}\right]}{3 A_{g}}
$$

where $A_{g}$ is the gross area of the cross section. $\left(A_{e}\right)$ and cross-section area. 


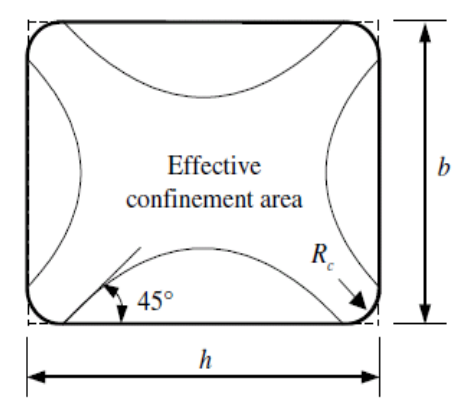

Figure 6: Effectively confined area.

The coefficient for taking into account the reduced effectiveness of FRCM compared to FRP is evaluated through the following analytical law:

$$
\begin{array}{ll}
k_{f l, F R C M}\left(\varepsilon_{c}\right)=1 & \text { for } \quad \varepsilon_{c} \leq 2 \varepsilon_{c 0} \\
k_{f l, F R C M}\left(\varepsilon_{c}\right)=\frac{1}{2} \frac{\varepsilon_{c} / \varepsilon_{c 0}}{\varepsilon_{c} / \varepsilon_{c 0}-1} & \text { for } \quad \varepsilon_{c}>2 \varepsilon_{c 0}
\end{array}
$$

which was found by Colajanni et al. [3] by fitting a series of experimental data of compression tests on concrete cylinders reinforced with two or three layers of PBO-FRCM.

\subsection{Constitutive models of materials}

The analytical model described above was adopted for generating the FE simulation of the compressive tests on the medium-size concrete specimens. The models were developed by means of the non-linear finite element code ABAQUS [5] using the Concrete Damaged Plasticity (CDP) model for the concrete material and properly adapted plastic parameters, according to some recent formulations available in the literature.

In particular, according to Hany et al. [1], the dilation angle $\psi$ and the coefficient $K_{c}$ were defined as:

$$
\begin{gathered}
\psi=-1.4587 \frac{A_{e}}{A_{c}} \frac{K_{l}}{f_{c 0}}+57.296 \\
K_{c}=\frac{5.5 f_{b 0}}{3 f_{c 0}+5 f_{b 0}}
\end{gathered}
$$

In Eqs. (7) and (8), $K_{l}$ is the stiffness of the jacket, $A_{e}$ and $A_{c}$ the effective and total cross-section area, respectively, and $f_{b 0}$ is initial equibiaxial compressive yield stress.

The dilation angle $\psi$ can be represented in the diagram of the flow potential hyperbolic function by Drucker and Prager, which is ruled by the equation:

$$
G=\sqrt{\left(\in \sigma_{t 0} \tan \psi\right)^{2}+\bar{q}^{2}}-\bar{p} \tan \psi
$$

The diagram of the Drucker and Prager flow potential function is reported in Fig. 7a.

The coefficient $K_{c}$ rules the shape of the yield surface; its graphic representation is reported in Fig. 7b, where the yield function by Lubliner et al. [6] is represented:

$$
F=\frac{1}{1-\alpha}\left(\bar{q}-3 \alpha \bar{p}+\beta\left(\tilde{\varepsilon}^{p l}\right)\left\langle\hat{\bar{\sigma}}_{\max }\right\rangle-\gamma\left\langle-\hat{\bar{\sigma}}_{\max }\right\rangle\right)-\bar{\sigma}_{c}\left(\tilde{\varepsilon}_{c}^{p l}\right)=0
$$




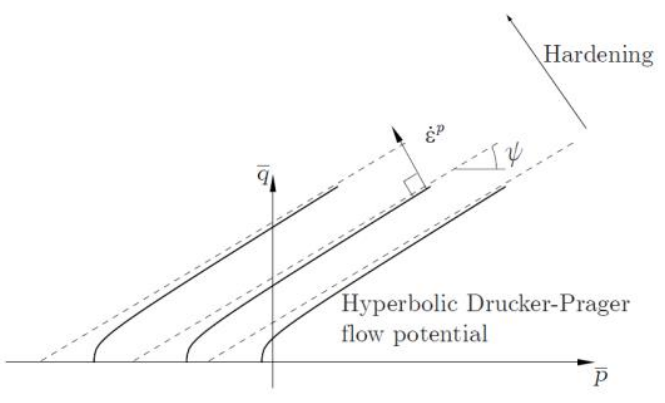

a)

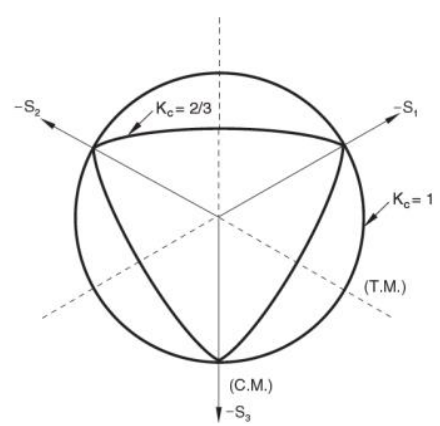

b)

Figure 7: a) Hyperbolic flow potential function; b) Yield function in the deviatoric plane [5].

The CDP model assumes isotropic damage of the material. The stress-strain law of compressed concrete (Fig. 8a) is given by Eq. (1) while, in tension, the input of the post-elastic behavior was given in terms of tensile strength $\sigma_{\mathrm{t} 0}$ and fracture energy $\mathrm{G}_{\mathrm{f}}$ (Fig. 8b).

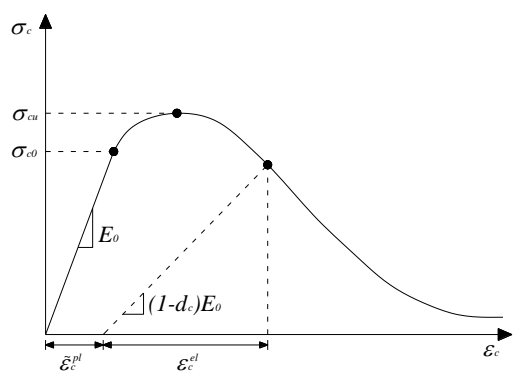

a)

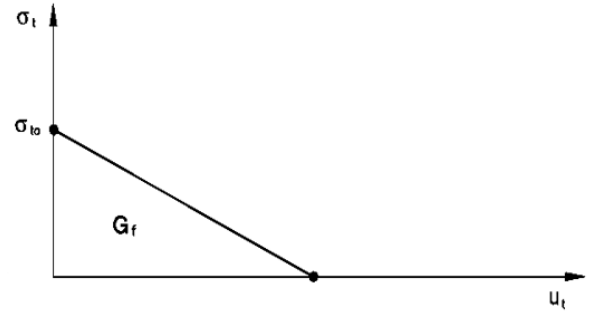

b)

Figure 8: Constitutive model of concrete: a) in compression; b) in tension.

For the simulation of the tests on the full-scale RC columns, also the steel reinforcement was modeled using a linear elastic behavior followed by a classical metal plasticity model where true stresses and logarithmic strains are implemented starting from the nominal values which can be obtained from a coupon tensile test:

$$
\begin{gathered}
\sigma_{\text {true }}=\sigma_{\text {nom }}\left(1+\varepsilon_{\text {nom }}\right) \\
\varepsilon_{\ln }^{p l}=\ln \left(1+\varepsilon_{\text {nom }}\right)-\frac{\sigma_{\text {true }}}{E}
\end{gathered}
$$

Finally, the steel concrete interface between concrete core and steel reinforcement was assumed perfectly bonded meaning that there is no modelling of the highly nonlinear stick-slip type of behavior of the interface with the rebars.

\subsection{Mesh and boundary conditions}

The geometry of the medium-size concrete specimens used for the simulation is shown in Fig. 9. In particular, a rectangular specimen is herein considered as an example. The specimen is $600 \mathrm{~mm}$ height and has a cross section of $200 \times 400 \mathrm{~mm}$ with corner radius equal to $15 \mathrm{~mm}$ (Fig. 9a). All degrees of freedom of the lower face of the specimen are constrained with an encastre and a reference point is individuated on the upper face where the compression is applied. The meshing technique adopted is structured and 8-node linear bricks with reduced integration are used, assuming a curvature control in the corner of the specimen that allow 16 elements per circle. The mesh size in the rest of the specimen is equal to $30 \mathrm{~mm}$. 


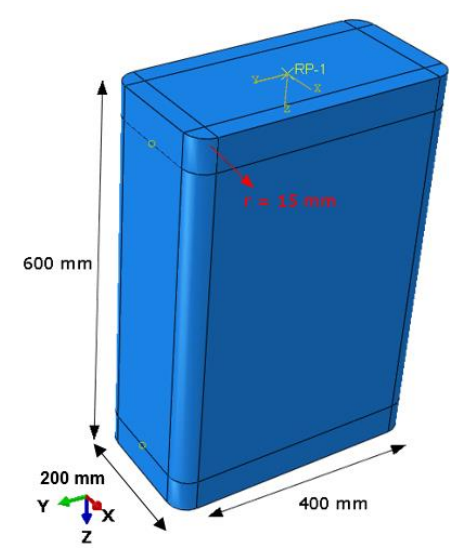

a)

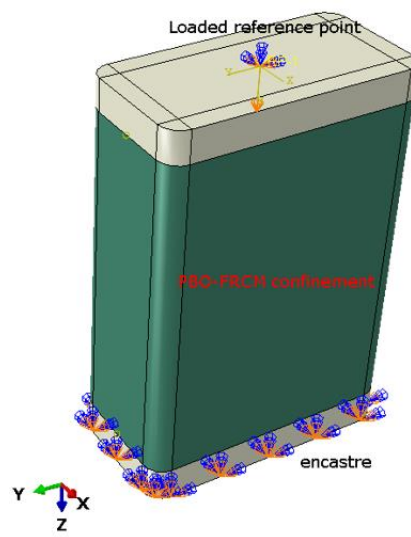

b)

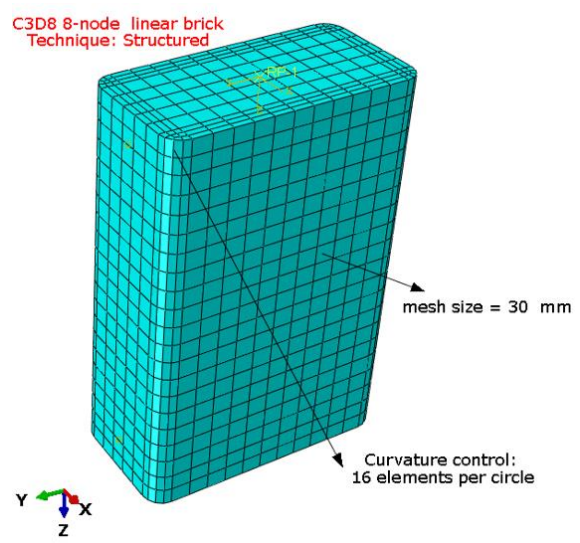

c)

Figure 9: Features of the FE concrete specimen: a) geometry; b) boundary conditions; c) mesh.

The FE specimen herein considered for the simulation of the full-scale RC column has rectangular cross-section 200x400 mm and corner radius $15 \mathrm{~mm}$ (Fig. 10a). It is wrapped with two layers of PBO-FRCM and subjected to a constant axial load of $500 \mathrm{kN}$. The columns is reinforced with six longitudinal rebars of steel class B450C and $16 \mathrm{~mm}$ diameter; moreover, transversal stirrups of $8 \mathrm{~mm}$ diameter are placed at a spacing of $170 \mathrm{~mm}$. The column is simply supported and loaded at the midspan, with shear span equal to $800 \mathrm{~mm}$ (Fig. 10b, Fig. 11).

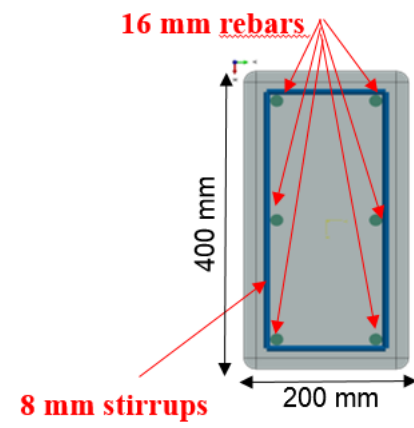

a)

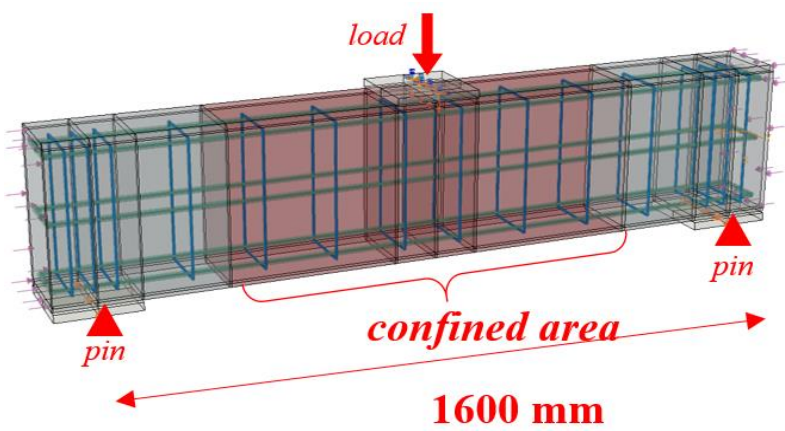

b)

Figure 10: Geometry of the FE column specimen: a) longitudinal scheme; b) cross-section.

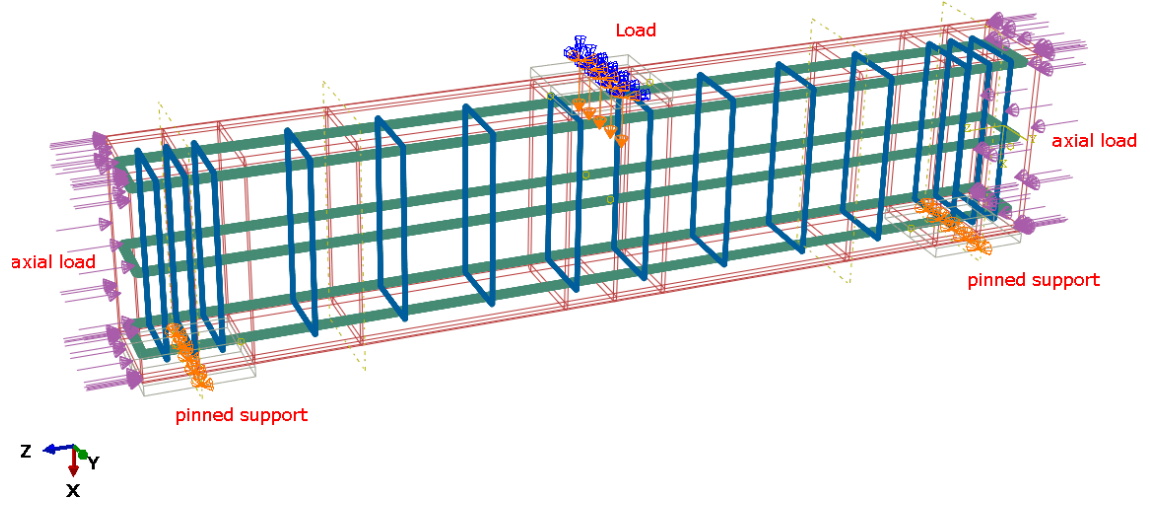

Figure 11: Load and boundary condition of the FE model of the RC column. 
The mesh used for the confined concrete material is constituted by 8 -node linear bricks with reduced integration and the meshing technique is structured thanks to the regularity of the geometry. Transversal stirrups and longitudinal rebar are meshed using 2-node linear beams (Fig. 12). The constraint between steel reinforcement and concrete is of "embedded" type, meaning that the bond splitting failure is prevented from occurring.

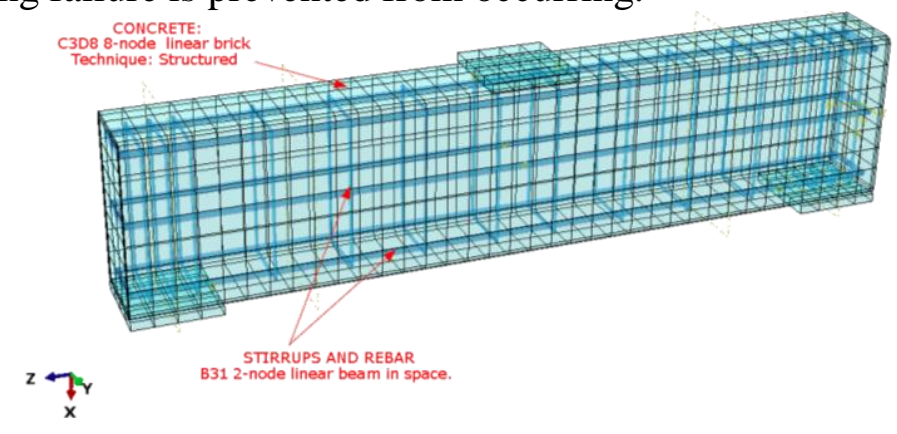

Figure 12: Mesh element types of RC column specimen.

\section{RESULTS}

Fig. 13a shows the comparison between the $f_{c^{-}} \varepsilon_{c}$ FE curve and the experimental curve of the rectangular specimen R19R30F2 whose features were already reported in Table 1. The same graph reports the analytical curve obtained from Eq. (1).

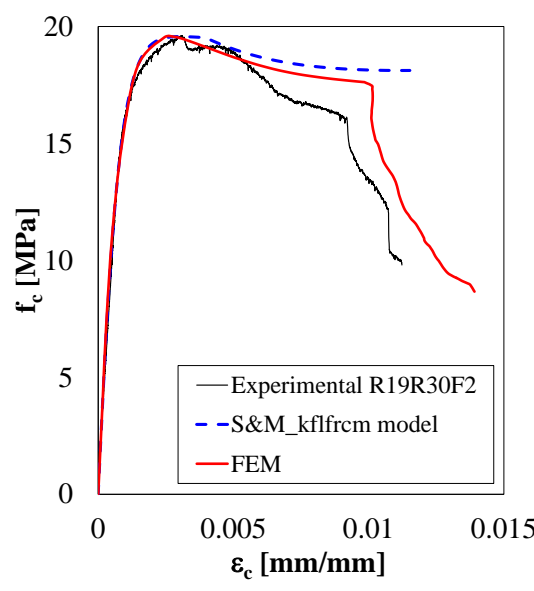

a)

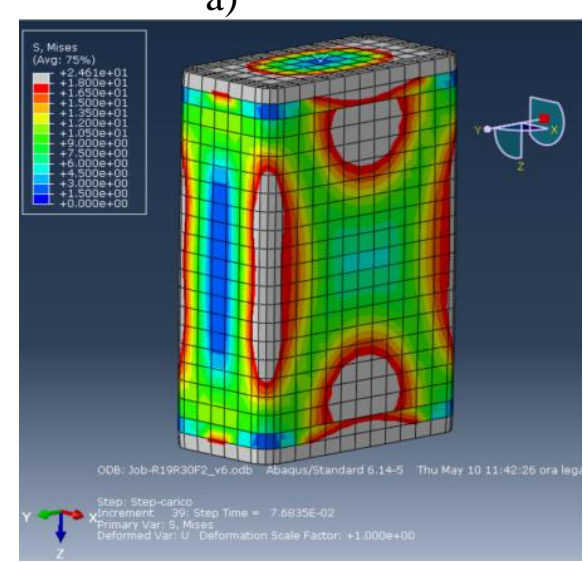

c)
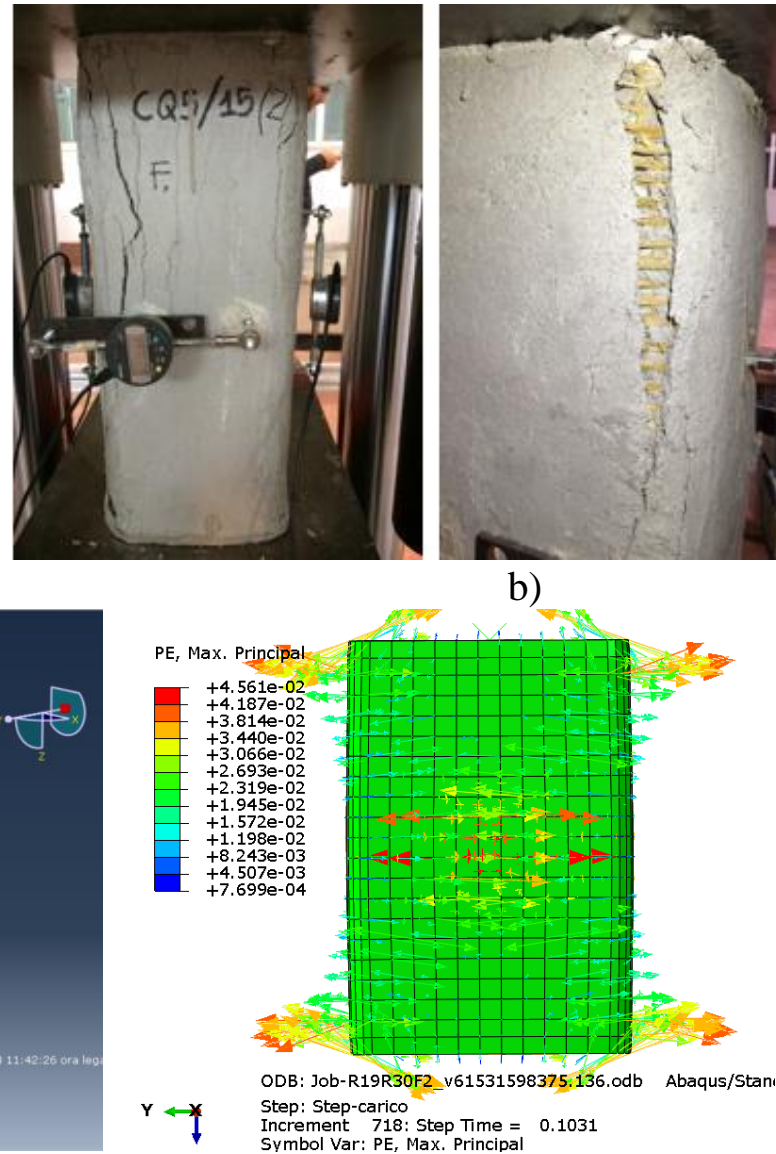

d)

Figure 13: Results of the FE model of concrete specimens: a) comparison between numerical, experimental and analytical curve; b) failure of the tested specimen; c) FE stress pattern; d) maximum principal strains. 
It can be observed that the implementation of the analytical model previously described allows reproducing the behavior in compression of the confined specimen obtained during the laboratory tests. Moreover, from the comparison between Fig. 13b and 13c it can be observed that the model is able to reproduce the actual failure mode characterized by the stress concentration mainly at the corners of the specimen which causes, firstly, the failure of the mortar and then the failure of the textile layers. Finally Fig. 13d depicts the pattern of the maximum principal strains in the post elastic regime and, therefore, it is able to show the FE crack pattern, compatible with the experimental evidence.

In Table 3 the results are summarized showing the peak stress for the specimen obtained in laboratory $\left(\mathrm{f}_{\mathrm{cc}, \mathrm{exp}}\right)$, with the FE simulation $\left(\mathrm{f}_{\mathrm{cc}, \mathrm{FEM}}\right)$ and from the analytical prediction $\left(\mathrm{f}_{\mathrm{cc} \text {, theo }}\right)$. Furthermore, the ratios between FE results and experimental and analytical model are reported showing a good agreement, the ratios being almost equal to one.

\begin{tabular}{|c|c|c|c|c|}
\hline$f_{c c, \exp }$ & $\mathrm{f}_{\mathrm{cc}, \mathrm{FEM}}$ & $f_{c c, \text { theo }}$ & $\mathrm{f}_{\mathrm{cc}, \mathrm{FEM}} / \mathrm{f}_{\mathrm{cc}, \mathrm{exp}}$ & $\mathrm{f}_{\mathrm{cc}, \text { theo }} / \mathrm{f}_{\mathrm{cc}, \exp }$ \\
\hline 19.615 & 19.554 & 19.567 & 0.997 & 0.998 \\
\hline
\end{tabular}

Table 3: Comparison between FE results, experimental data and analytical prediction.

The FE model can be considered validated and can be used for the more complex simulation of the behavior of confined RC columns. The unconfined specimen RC19R15F0 and the specimen confined with two layers RC19R15F2 were considered. Their main features were already reported in Table 2. The comparison between numerical and experimental load-midspan deflection curves is shown in Fig. 14 for both unconfined and confined specimens. The model shows a quite good prediction of the behavior of the confined specimen even though it is not able to catch the softening branch before the failure of the specimen when the ultimate displacement is attained. As regards the simulation of the unconfined specimen, the model shows a good capacity in the prediction of the peak load but it is not suitable in catching the large plastic displacement regime before failure.

The stress state obtained through the FE model is depicted in Fig. 15 for the confined concrete (Fig. 15a) and for the steel reinforcement (Fig. 15b). In particular, comparing Fig. 15a and the pictures in Fig. 15c, it can be observed that the model is able of reproducing the crack opening on the bottom of the specimen before failure. It can also be observed the stress concentration in correspondence of the limits of the application of the PBO-FRCM reinforcement. Finally the stresses in the steel rebars prove to be compatible with the experimental evidence.

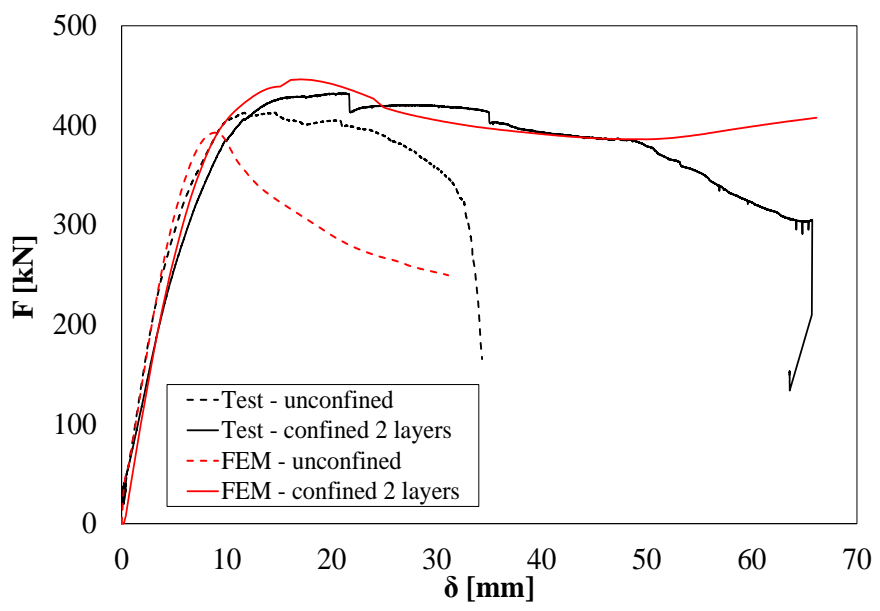

Figure 14: Load-deflection curve of the full-scale RC column: numerical vs. experimental results. 
Table 4 reports the comparison between numerical and experimental results in terms of max-

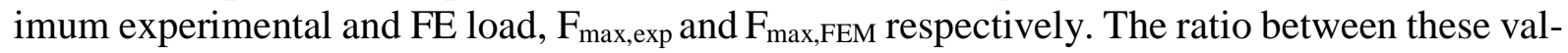
ues is equal to 0.94 for the unconfined specimen and 1.04 for the confined columns, showing a good agreement with the laboratory tests in terms of maximum capacity of the RC specimen.
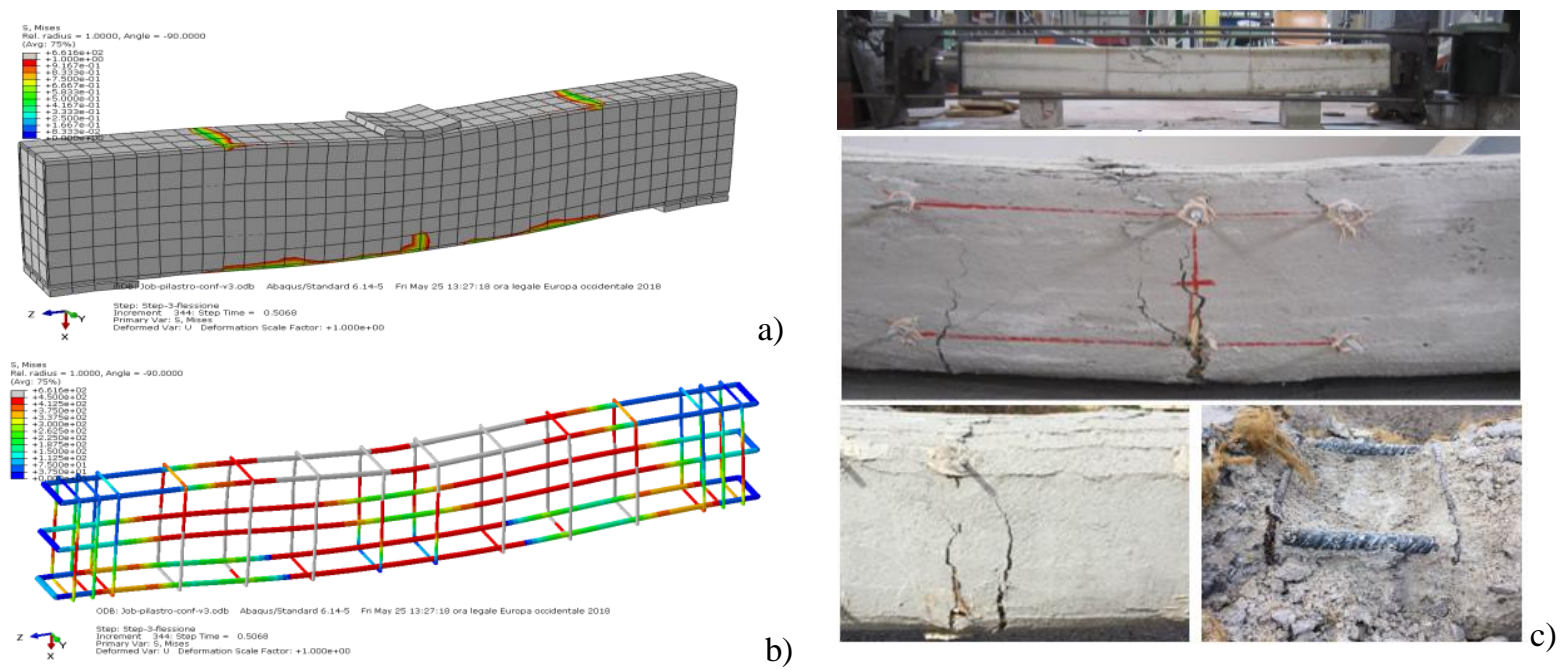

b)

Figure 15: Results of the FE model of the RC columns: a) stress pattern in the confined concrete; b) stresses in the steel reinforcement after the yielding; c) experimental failure and crack pattern.

\begin{tabular}{cccc}
\hline ID & $F_{\text {max,exp }}$ & $F_{\text {max,FEM }}$ & $F_{\text {max,FEM }} / F_{\text {max } \text { exp }}$ \\
\cline { 5 - 5 } RC19R15F0 & 418.06 & 392.58 & 0.94 \\
RC19R15F2 & 438.44 & 455.62 & 1.04 \\
\hline
\end{tabular}

Table 4: Comparison between FE results and experimental data of the RC columns.

\section{CONCLUSIONS}

- The seismic performance of existing buildings is strongly restricted by both low strength of concrete and poor detailing of transverse reinforcement in columns. Confinement by thin steel plates and angles welded to the corners of beams and columns or the jacketing by FRP are widespread efficient techniques for increasing strength and ductility of structural elements especially of columns but recently some drawbacks in the use of FRP have been recognized: they cannot be applied with epoxy resin on moist elements, exhibit poor performance in the presence of heat and fire and lack of vapour permeability; the hardening phase is strongly affected by environmental temperature; in some cases the epoxy resin is not compatible with the concrete substrate.

- In this framework, PBO-FRCM is an effective tool for increasing strength and ductility of low strength under-detailed concrete columns of seismic resistant frames.

- A FE model is developed for simulating the behavior of RC squared and rectangular columns strengthened by PBO-FRCM, under axial force and bending moment. The load condition of columns in MRFs loaded by horizontal forces with constant axial load is reproduced. Tests on large scale specimens confined with PBO-FRCM are used for the validation of the FE model. 
- The modeling technique is preliminarily validated developing FE models of specimens of plain concrete wrapped by PBO-FRCM and the results are validated against experimental data.

- The FE model used is able to predict both strength and displacement increment as well as the local strength degradation in the post peak range.

\section{REFERENCES}

[1] N.F. Hany, E.G. Hantouche, M.H. Harajli, Finite element modeling of FRP-confined concrete using modified concrete damaged plasticity. Eng. Struct., 125,1-14, 2016.

[2] M.R. Spoelstra, G. Monti, FRP confined concrete model. J. of Compos. For Constr., 3(3), 143-150, 1999.

[3] P. Colajanni, F. De Domenico, A. Recupero, N. Spinella, Concrete columns confined with fiber reinforced cementitious mortars: experimentation and modelling. Constr. and Build. Mat., 52, 375-84, 2014.

[4] CEB-FIP Bullettin $n^{\circ} 14$. Externally bonded FRP reinforcement for RC structures; 2001.

[5] Abaqus/CAE User's Manual, v. 6.14, 2014.

[6] L. Lam, J.G. Teng, Design-oriented stress-strain model for FRP confined concrete. Constr. Build. Mater. 17(6,7), 471-489, 2003.

[7] L. Lam, J.G. Teng. Ultimate condition of fiber reinforced polymer-confined concrete. $J$ Compos Constr. 8(6), 539-48, 2004.

[8] L. Lam, J.G. Teng. Stress-strain model for FRP-confined concrete under cyclic axial compression. Eng Struct. 31(2), 308-21, 2009.

[9] Z. Wang, D. Wang, S. Smith, D. Lu. CFRP-confined square RC columns. II: Cyclic axial compression stress-strain model. J Compos Constr. 16(2), 161-70, 2012.

[10] Z. Wang, D. Wang, S. Smith, D. Lu. Experimental testing and analytical modeling of CFRP-confined large circular RC columns subjected to cyclic axial compression. Eng Struct. 40, 64-74, 2012.

[11] N. Nisticò, F. Pallini, T. Rousakis, Y.F. Wu, A. Karabinis. Peak strength and ultimate strain prediction for FRP confined square and circular concrete sections. Composites $B$. 67, 543-54, 2014.

[12] N. Hany, E. Hantouche, M. Harajli. Axial stress-strain model of CFRP confined concrete under monotonic and cyclic loading. J Compos Constr. 19(6), 04015004, 2015.

[13] C. Pellegrino, D. Tinazzi, C. Modena. An experimental study on bond behavior between concrete and FRP reinforcement. J Compos Constr. 12(2); 180-9, 2008.

[14] A. Mirmiran, K. Zagers, W.Q. Yuan. Nonlinear finite element modeling of concrete confined by fiber composites. Finite Elem Anal Des. 35(1), 79-96, 2000.

[15] S.H. Lo, A.K.H. Kwan, Y. Ouyang, J.C.M. Ho. Finite element analysis of axially loaded FRP-confined rectangular concrete columns. Eng Struct. 100, 253-63, 2015. 